\title{
Cannabis and cannabinoids: pharmacology and therapeutic potential
}

\author{
Małgorzata Śmiarowska ${ }^{1}$, Monika Białecka ${ }^{1}$, Anna Machoy-Mokrzyńska ${ }^{2}$ \\ ${ }^{1}$ Department of Pharmacokinetics and Therapeutic Drug Monitoring, Pomeranian Medical University, Szczecin, Poland \\ ${ }^{2}$ Department of Experimental and Clinical Pharmacology, Pomeranian Medical University, Szczecin, Poland
}

\begin{abstract}
Introduction. Cannabis (also known as marijuana) is the most frequently used psychoactive substance in the world. The role of cannabis in medicine is rapidly evolving, and advances in the understanding of its pharmacology have led to numerous proposed uses of these drugs.

State of the art. Cannabis contains $\Delta 9$-tetrahydrocannabinol and cannabidiol as the primary constituents responsible for pharmacological activity. It is now known that there are at least two types of cannabinoid receptors. CB1 receptors are found mainly in the CNS, and their primary role is to inhibit the release of neurotransmitters. CB2 receptors'leading role is to modulate cytokine release and immune cell migration. Colocalisation of cannabinoid receptors with other types of nervous system receptors allows them to interact with many other transmitters such as dopamine, noradrenaline, acetylcholine, gamma-aminobutyric acid, serotonin, and glutamic and aspartic acids.

Clinical implications. The rapidly expanding understanding regarding cannabinoids led to initial attempts to treat selected diseases with cannabinoid receptor agonists and antagonists. The most promising of these was the potential possibility of treating diseases for which current therapy is unsatisfactory, such as neurological diseases including multiple sclerosis, spastic muscular tension, extrapyramidal system diseases, neurodegenerative diseases and cerebral ischaemia. Attempts to treat psychiatric diseases (e.g. psychoses, neuroses, mood disorders, and alcohol dependence syndrome) with cannabinoids are much less advanced.

Future directions. Cannabis and cannabinoids can be widely used to treat several diseases or alleviate symptoms, but their efficacy for specific indications is not always apparent. Further exploration is needed to understand whether the enhanced sensitivity to the cognitive effects of $\triangle 9$-THC depends on brain cannabinoid receptor dysfunction, and how these changes contribute to the cognitive deterioration and core pathophysiology symptoms associated with schizophrenia or other neurological and somatoform disorders.
\end{abstract}

Key words: cannabis, cannabinoids, THC, cannabinoid receptors

(Neurol Neurochir Pol 2022; 56 (1): 4-13)

\section{Definition of cannabis and cannabinoids}

Cannabis is commonly known as marijuana. This plant extract is the most frequently used psychoactive vegetal substance in the world. It is obtained from Cannabis sativa or Cannabis indica. It contains over 500 ingredients, of which more than 100 active substances have been identified, including $\Delta$ 9-tetrahydrocannabinol ( $\Delta 9$-THC), cannabidiol (CBD), cannabigerol, cannabidiol acid, cannabicyclol, cannabichromene, and cannabinol $[1,2]$. Cannabis potency is evaluated in terms of the ratio between the two primary psychoactive components: $\triangle 9$-THC to cannabidiol CBD. The

Address for correspondence: Monika Białecka, Department of Pharmacokinetics and Therapeutic Drug Monitoring, Pomeranian Medical University, Szczecin, Poland; e-mail: monika-bialecka@post.pl

Received: 29.11.2021 Accepted: 31.01.2022 Early publication date: 8.02.2022

This article is available in open access under Creative Common Attribution-Non-Commercial-No Derivatives 4.0 International (CC BY-NC-ND 4.0) license, allowing to download articles and share them with others as long as they credit the authors and the publisher, but without permission to change them in any way or use them commercially. 
variable composition of the raw cannabis plant, especially the varying $\triangle 9$-THC/CBD ratios, renders cannabis therapeutic while also being associated with detrimental effects [3]. The term 'cannabinoids' refers to every chemical substance, regardless of structure or origin, that joins the cannabinoid receptors, so they constitute ligands for these receptors. They are a group of terpenophenolic compounds found in cannabis, characterised by a tricyclic structure and benzopyran derivatives with 21 carbon atoms.

\section{Cannabinoids receptors}

Cannabinoids produce their effects by activating two different $G$ protein-coupled receptors (GPCRs) identified as cannabinoid receptors known as metabotropic because they involve changes in the cell's metabolism and differences in the activation of specific second messenger systems. The activation of $G$ proteins leads to increased synthesis of cyclic AMP, resulting in the activation of cyclic AMP-dependent kinase (PKA), which phosphorylates a channel protein. These result in a modification in ionic permeability.

According to the International Pharmacological Union and Cannabinoid Receptors Subcommittee, cannabinoids receptors are divided into receptor subtypes [4]. The first subtype of cannabinoid receptors was sequenced, cloned, and named CB1. This is encoded by the CNR1 gene and its gene locus is chromosome 6 at the 6p14-q15 location. This receptor consists of 472 amino acids weighing about $64 \mathrm{kDa}$. These receptors are found mainly in the central nervous system (CNS), and their primary role is to inhibit the release of neurotransmitters. They are highly expressed in the brain, and mediate most of the significant effects of cannabis. The activation of CB1 results in the inhibition of adenyl cyclase activity and a consequent decrease in cytosolic cAMP content, closure of voltage-gating $\mathrm{Ca}^{2+}$ channels, the inward opening of $\mathrm{K}^{+}$channels, and the stimulation of kinases, which phosphorylate tyrosine, serine, and threonine residues in proteins.

The CB1s are among the most abundant GPCRs on neurons in the CNS, and their expression pattern is consistent with the psychoactive and psychomotor effects of cannabis. High levels of CB1s are found in brain regions including the cortex, forebrain, limbic system (hippocampus, hypothalamus, amygdala), striatum (basal ganglia, nucleus accumbens, substantia nigra, globus pallidus), and cerebellum. In other words, the regions responsible for memory, sleep and wakefulness, emotions, coordination of movements, and body posture $[5,6]$. The analgetic property of $\mathrm{CB} 1 \mathrm{~s}$ relates to the pain pathways containing the periaqueductal grey matter, afferent spinal cord regions, peripheral nociceptors, and spinal interneurons [7]. In contrast, relatively low levels of CB1s are found in other medulla/brainstem nuclei (an exception is the brainstem emetic centre), such as those whose areas are involved in controlling respiration (a feature that distinguishes $\mathrm{CB} 1$ from opiate receptors) and the thalamus responsible for essential life processes [5].
Beyond the CNS, CB1s are also widely expressed in other peripheral organs such as the heart, lungs, gastrointestinal tract, kidneys, bladder, uterus, placenta, testicles, vas deferens, skeletal muscle, bone, joints, skin, and even in cells of the immune system [6].

The second cannabinoid receptor subtype to be discovered, abbreviated as $\mathrm{CB} 2$, was initially thought to have been peripheral and not to mediate any central effects of THC. This cannabinoid receptor is also $\mathrm{G}$ protein-coupled which is both positively and negatively coupled to the mitogen-activated protein kinase (MAPK) and CAMP pathways [7]. CB2 receptors are encoded by the CNR2 gene located on chromosome $1 \mathrm{p} 36$. CB2 receptors are primarily localised in the immune system: the tonsils, spleen, and in various immune system cell lines, especially on lymphocytes, natural killer cells (NK), and on monocytes, macrophages, mast cells, and microglial cells [8]. CB2s appear to be most concentrated in tissues and immune system cells such as the leukocytes and spleen. Their chief role is to modulate cytokine release and immune cell migration. They are also present at low levels in several brain areas; they are especially expressed in microglia, blood vessels, and some neurons. CB2 receptors are expressed at much lower levels in the CNS compared to CB1.

The distribution of CB1 and CB2 receptors has been determined using various techniques, such as ligand attachment studies to the receptor, the use of antibodies against the respective receptor types, autoradiography, Northern Blotting, the in situ hybridisation technique, and Real-Time PCR. By comparing the transmembrane domains of the $\mathrm{CB} 1$ receptor with the $\mathrm{CB} 2$ receptor, $44-66 \%$ homology has been demonstrated. However, CB2 does appear to be expressed by some neurons, particularly under certain pathological conditions [9]. The $\mathrm{CB} 1$ and $\mathrm{CB} 2$ receptors central and peripheral dissemination and accessibility are essential for their possible therapeutic use and side effects when the synthetic cannabinoids (SCs) are supposed to be adhibited [10,11].

\section{Cannabinoids receptors ligands}

Plant-derived compounds, i.e. tetrahydrocannabinols and synthetic functional analogues with a different chemical structure (so-called 'synthetic cannabinoids'), can be ligands for cannabinoid receptors. To date, they have mainly gained popularity as substitutes for natural tetrahydrocannabinols which are under legal control. They have been referred to as 'legal highs' or 'designer drugs'. Endogenous compounds physiologically present in the human body called endocannabinoids are also ligands for cannabinoid receptors.

In 1992, the first such endogenous compound was identified and named anandamide. Endocannabinoids were found to be derivatives of omega- 6 fatty acids. The best-studied endogenous cannabinoids include amide, ester, or ether derivatives of arachidonic acid. These include arachidonoyl ethanolamide (AEA), 2-arachidonyl glycerol (2-AG), noladin 
ether (arachidonoyl glycerol ether), virodhamine (an ester of arachidonic acid with ethanolamine), or $\mathrm{N}$-arachidonoyl dopamine (NADA). These compounds differ in their affinity for both types of cannabinoid receptors, the strength of their agonist action, and the stability of their structure. They are produced 'on demand' in the postsynaptic membrane of the neuron from phospholipid precursors and are characterised by local action, meaning that they are not stored and are immediately degraded after having produced an appropriate biological effect. Studies confirm that endocannabinoids act as so-called retrograde transmitters [12], which means that they diffuse through the synaptic gap to the cannabinoid receptors located in the presynaptic membrane and inhibit the release of neurotransmitters. Colocalisation of cannabinoid receptors with other types of nervous system receptors allows them to interact with many transmitters such as dopamine, noradrenaline, acetylcholine, gamma-aminobutyric acid, serotonin, glutamic and aspartic acids and others [7].

Endocannabinoids play an important, yet still not fully understood, biological role consisting mainly in the regulation of many physiological processes. Large-scale studies have demonstrated their involvement in antinociception, i.e. inhibition of pain [6], learning and memory processes, control of emotional states, the pathogenesis of schizophrenia, neurodegenerative diseases, in the phenomenon of neuroprotection, as well as in food intake regulation, hormone secretion of the hypothalamic-pituitary-adrenal axis, modulation of immunological processes, inflammatory reactions, cell proliferation, and apoptosis [13].

\section{Endocannabinoid system (ECS)}

Cannabinoid receptor ligands, $\mathrm{CB} 1$ and $\mathrm{CB} 2$ receptors, and enzymes involved in endocannabinoid synthesis and degradation are part of an important signalling system known as the endocannabinoid system (ECS) or endocannabinoid physiological control system [14]. There is ample evidence supporting a role for the ECS in the neuromodulation of all neurotransmitters (both excitatory and inhibitory) involved in the reward system, which is supposed to be related to the development of addiction.

Although CB1 and CB2 receptor ligands hold the greatest promise for their potential therapeutic use, there are also concerns that they may be used for non-medical indications. To date, intensive research has categorised cannabinoid receptor agonists into four classes.

The first class consists of dibenzopyran derivatives, namely delta-9- and delta-8-tetrahydrocannabinols. The second class includes more potent compounds than THC, i.e. synthetic dicyclic compounds with analgesic effects. The third class is assigned to agonists - with an amino-alkyl-indole structure exhibiting anti-inflammatory activity. The fourth class includes endogenous cannabinoids, attributed to anti- or pro-apoptotic effects, depending on the cell type, maturity, and cannabinoid properties.
The apoptotic function of endocannabinoids has been demonstrated in studies on glioma, breast, and prostate cancer cell lines. Considerable research has been devoted to the neuroprotective effects of cannabinoids, which have now been clinically confirmed. There have been attempts to use them in the treatment and prevention of stroke and the treatment of craniocerebral trauma. Their action is related to the inhibition of glutamic acid release; however, antagonism to the NMDA receptor, antioxidant properties, inhibition of nitric oxide release from microglia cells, and blockade of calcium channels also appear to be important [7].

It is also known that ECS is an ancient, evolutionarily conserved, ubiquitous lipid signalling system active in preand postnatal life. It is a widespread neuromodulatory system in CNS. Recent studies have highlighted an essential role of endocannabinoid signalling (ECBS) in nervous system development, including neurogenesis (axon guidance and synaptic plasticity, neuronal migration), which is crucial to achieving the complex architecture and efficient wiring of the CNS [8]. Endocannabinoids are arachidonic acid derivatives synthesised from membrane phospholipid precursors in response to cellular requirements (e.g. in response to an action potential in neurons or another type of biological stimulus). It appears that there may be a certain amount of cross-talk between the eicosanoid and endocannabinoid pathways [6]. The endocannabinoids are synthesised during periods of intense neuronal activity [9].

Therefore, ECBS is involved in a great many physiological and pathophysiological central and peripheral processes determining cognitive processes (learning, memory), regulation of stress, emotional state/mood and behaviour, psychomotor activity, immune function, inflammation, pain, wake/sleep cycles and appetite regulation, metabolism, and energy expenditure, homeostasis, cardiovascular and digestive function, bone development and density, and reproduction $[1,6,15,16]$.

Furthermore, there is strong evidence that dysregulation of the ECBS contributes to many pathological conditions including pain, inflammation, psychiatric disturbances, and neurodegenerative processes $[6,10,14]$. The biological function of ECS is mediated by the cannabinoid receptors CB1s and $\mathrm{CB} 2 \mathrm{~s}$, which are widely diffused in many tissues of the body, as described above $[1,2]$.

\section{Therapeutic applications of cannabinoids}

Despite the rapidly expanding knowledge regarding cannabinoids, it was not until the late 20th century that attempts were made to treat selected diseases with cannabinoid receptor agonists and antagonists. The most exciting of all was the potential possibility of treating diseases for which current therapy is unsatisfactory. These include neurological diseases such as multiple sclerosis, spastic muscular tension, extrapyramidal system diseases or neurodegenerative diseases (e.g. Parkinson's Disease or Tourette's Syndrome), and cerebral ischaemia. 
Table 1. Comparison of most commonly used cannabinoids: $\triangle 9-\mathrm{THC}$ and CBD [3-9]

\begin{tabular}{|c|c|c|}
\hline & $\Delta 9-T H C$ ( $\Delta 9$-tetrahydrocannabinol) & CBD (cannabidiol) \\
\hline $\begin{array}{l}\mathrm{CB} 1 \text { and } \mathrm{CB} 2 \\
\text { affinity }\end{array}$ & Partial agonist CB1 and CB2 & $\begin{array}{l}\text { Poor bond with } \mathrm{CB} 1 \text { and } \mathrm{CB} 2 \text {, } \\
\text { possible antagonist }\end{array}$ \\
\hline \multirow{8}{*}{$\begin{array}{l}\text { Other receptors } \\
\text { interaction }\end{array}$} & No interaction with TRPV1 & TRPV1 agonist \\
\hline & TRPV2 agonist & TRPV2 agonist \\
\hline & TRPA1 agonist & TRPA1 agonist \\
\hline & TRPM8 antagonist & TRPM8 antagonist \\
\hline & GPR55 agonist & GPR55 antagonist \\
\hline & 5- $\mathrm{HT}_{3 \mathrm{~A}}$ antagonist & 5- $\mathrm{HT}_{1 \mathrm{~A}}$ agonist \\
\hline & PPAR $y$ agonist & PPARy agonist \\
\hline & Positive allosteric effector of glycine receptors & $\begin{array}{l}\text { Positive allosteric effector of glycine } \\
\text { receptors }\end{array}$ \\
\hline \multirow[t]{7}{*}{ Expected effect } & Analgetic & Anticonvulsive \\
\hline & Flaccidity & Flaccidity \\
\hline & Anti-vomiting & Anti-anxiety \\
\hline & Appetite analeptic & Anti-inflammation \\
\hline & Psychoactive & Neuroprotective \\
\hline & & Anti-oxidative \\
\hline & & Anti-psychotic \\
\hline Possible tolerance & $\begin{array}{l}\text { Pharmacodynamic tolerance: desensitisation and CB1 down-regulation; except } \\
\text { analgetic antispastic action; a pharmacodynamic tolerance of low level is possible }\end{array}$ & Has not been proved \\
\hline
\end{tabular}

Attempts to treat psychiatric diseases (e.g. psychoses, neuroses, mood disorders, and alcohol dependence syndrome) with cannabinoids are much less advanced $[17,18]$.

The difficulties arise from potential psychoactive effects and the inability to account for these compounds' effects on impaired neurotransmission. In internal medicine diseases, attempts have been made to exploit cannabinoids' hypotensive, bronchodilatory, intraocular pressure-lowering, and most importantly, antiemetic, anti-inflammatory, and analgesic effects. Cannabinoids alleviate acute and chronic pain of various aetiologies, including postoperative, cancer, migraine, neuropathic, and rheumatic pain. Since the psychoactive effects of these compounds are a concern, new opportunities have arisen in the search for selective agonists that will exhibit analgesic effects but lack adverse central effects $[2,14,19]$. Based on studies on the role of cannabinoids in regulating food intake, attempts have been made to use ligands for cannabinoid receptors to treat anorexia in cancer or AIDS patients. Cannabinoids have attracted some interest due to their therapeutic activity in resistant epilepsy, addictions, psychotic disorders, anxiety, and sleep disorders [11]. This is possible because of the central location of cannabinoids receptors and their interaction with primary neurotransmitters.

There are a few sublime pharmacodynamic differences between these two main phytocannabinoids $\triangle 9$-THC and $\mathrm{CBN}$. It has been proved that $\triangle 9-\mathrm{THC}$ is a partial agonist of both $\mathrm{CB} 1$ and $\mathrm{CB} 2$ receptors, and their affinity is responsible not only for analgetic, anti-inflammatory, and anti-vomiting action, but also for the psychoactive effect (Tab. 1).
Synthetic cannabinoids (SCs), as with natural cannabis, have been the subject of great interest in recent years. So far, the therapeutic value of numerous SCs has been documented [20]. The second generation of synthetic cannabinoid receptor agonists, particularly $\mathrm{CB} 1 / \mathrm{CB} 2$ receptors agonists that do not readily cross the blood-brain-barrier, and a large and ever-growing population of $\mathrm{CB} 2$ receptor-selective ligands, are both thought to possess potential therapeutic applications, at least for the management of inflammatory, neuropathic and cancer pain, as they give better benefit-to-risk ratios than centrally active $\mathrm{CB} 1$ receptor agonists $[7,20]$.

A systematic review and meta-analysis has shown that there is one formulated drug consisting of whole plant extract from the Cannabis species Cannabis sativa $L$. that has been purified into the active components: cannabidiol (CBD), nabilone, and dronabinol (synthetic analogues of $\triangle 9$-THC), nabiximol (a combination of synthetic $\Delta 9-\mathrm{THC}$ and CBD in equal 1:1 proportions). For trademark purposes, these purified products are branded as Nabidiolex ${ }^{\circledR}$, Tetrabinex ${ }^{\circledR}$, and Sativex ${ }^{\circledR}$, respectively. The USA Food and Drug Administration (FDA) has approved them in the treatment of spasticity in multiple sclerosis, in cases of chronic cancer, spastic and neuropathic pain, in patients suffering from anorexia due to HIV/AIDS, in Tourette's Syndrome, and as a second-line treatment in nausea and vomiting induced by cancer chemotherapies $[6,11]$. These products seem to have always been the most frequently studied and medically preactivated cannabinoid forms available in Europe. For example, Sativex ${ }^{\circledR}$ is available in Europe as an oro-mucosal pump spray used in restricted indications 
for the relief of moderate to severe spasticity symptoms in adult multiple sclerosis (MS) patients who are unresponsive to other spasticity-alleviating medicinal products and who show a clinically significant improvement in spasticity-related symptoms in the initial stage of therapy.

Most clinical studies have shown symptom improvement after cannabinoid application, but the results have not been statistically significant. Thus, the evidence confirming their therapeutic value is of moderate quality for chronic pain and spasticity and of low quality in nausea and vomiting due to chemotherapy, weight gain in HIV, sleep disorders, and Tourette's Syndrome [11, 17]. Synthetic cannabinoids increase dopamine (DA) levels and potentiate DA-expressing neurons. Thus, the ability of cannabinoid agonists to increase DA concentration implicates pathways where the ECS is involved in stress and reward regulation [21]. It may be the case that SCBs strengthen the pro-psychotic effects via upregulation of the 5-HT2A receptors in a brain region-specific manner.

On the other hand, 5-HT2A receptors agonists and antagonists produce contradictory psychosis-like results observed in the head-twitch response (HTR) compared to the cannabinoid receptor agonists and antagonists. The interaction between CB1 signalling and 5-HT2A receptor expression and function is still under research. It is thought that the interaction between SCBs and brain serotonin systems depends critically on the dose, frequency of administration, or other procedural factors [21]. It has been demonstrated in animals that cannabinoid compounds strongly influence memory extinction and impair memory retrieval. These observations have facilitated the successful treatment of patients with post-traumatic stress disorder (PTSD) with synthetic cannabinoids. The cannabinoid receptor agonist nabilone has been shown to have a therapeutic effect on treatment-resistant nightmares in patients with PTSD, with some patients reporting subjective improvements in sleep duration and quality, and fewer daytime flashbacks [15].

In addition to naturally-derived cannabinoids, their synthetic counterparts (nabilone, dronabinol) and other substances with different chemical structures (such as WIN $55,212-2$ ) with high affinity for CB1 and CB2, receptors are used in research as well as in medical practice.

The evidence from controlled clinical trials suggests that cannabinoids relieve the symptoms of some illnesses, mainly because in many neurological diseases, neurotransmission abnormalities lead to overexcitation or lack of inhibition due to cannabinoid receptors [17]. However, they are often used as adjunctive treatments, which means that they are only introduced when a patient fails to respond to standard treatments.

\section{Neuroprotective aspects of cannabinoids}

Many studies have focused on cannabinoids' (CBs) neuroprotective role. This complex process is composed of the $\mathrm{CB}$ anti-oxygenating properties, inhibiting $\mathrm{NO}^{*}$ release from microglia cells, subsequent blockade of calcium channels [22], and $\gamma$-aminobutyric acid (GABA) release [9]. The principal protective value seems to have $\mathrm{CBs}$ and glutamate (Glu) connection. Glutamate is a fundamental excitatory neurotransmitter in the CNS, playing critical roles in memory, neuronal development, and synaptic plasticity. Moreover, excessive glutamate release has been linked to neuronal cell death. Glutamate binds to both ionotropic and metabotropic receptors (mGluR). There is scant data on interactions between $\mathrm{mGluR}$ and $\mathrm{CB}$ receptors. Activation of NMDA receptors can induce endocannabinoid release from postsynaptic neurons. Subsequently, endocannabinoids can decrease the activity of NMDA receptors. It is clinically essential that the NMDA receptors are most closely associated with psychiatry and neurological disorders, including schizophrenia and psychosis. Multiple mechanisms may allow cannabinoids to modulate NMDA receptor activity. Depolarisation-induced CB1 activation may influence glutamate inhibition [22] and events downstream from CB1R signalling, which may attenuate NMDA receptor signalling. It is possible that synthetic cannabinoids displaying significant antagonist affinity at the NMDA site would be more likely to induce pro-psychotic effects in human users [15].

Preclinical studies evaluating the effects of cannabinoid receptor blockade or activation on emotional responses have produced somewhat contradictory results. However, the consensus is that endocannabinoids play a crucial role in maintaining emotional homeostasis [22]. The endogenous cannabinoids-mediated control of relevant brain regions (mainly the hippocampus, prefrontal cortex, amygdala, and hypothalamus) and their interregional connectivity with the hypothalamic-pituitary-adrenal axis and sympathetic nervous system have been suggested to participate in anxiety, fear, and stress-coping [23], like astroglial CB1-dependent mechanisms that operate in the effect of cannabinoids and endocannabinoids signalling on these processes. The role of the $\mathrm{CB} 1 \mathrm{~s}$ expressed at presynaptic terminals seems to reduce neurotransmitter release, while the astroglial $\mathrm{CB} 1$ appears to potentiate glutamatergic synaptic signalling in emotional regulation [23].

In more recent years, studies on endocannabinoids (AEA, 2-AG) have suggested that metabotropically induced suppression of inhibition (or excitation) is a ubiquitous form of short-term or long-term synaptic plasticity mediated by endocannabinoids $[9,24]$. This phenomenon is referred to as 'endocannabinoid-mediated plasticity' because the mechanism appears to occur at multiple synapses throughout the CNS, spreading from the cortex to the spinal cord $[5,9,24]$. Cannabinoids, through their receptors, can modulate the activity of multiple cell types (neuronal subtypes, astrocytes, and microglia), and in turn, can execute context-related alterations in synaptic transmission, resulting in fine-tuned patterns of neuronal activity [23].

To conclude, it should be noted that these neuroprotective aspects of cannabinoids have been observed and determined 
mainly in vitro and based on animal experiments. To date, clinical evidence of their neuroprotection in humans has yet to be obtained, and preclinical studies are still necessary to confirm this prior to practical use.

\section{Potential hazards of cannabinoids}

An increased risk of short-term adverse effects (AEs) requires consideration while discussing cannabinoids therapy. The common AEs include dizziness, dry mouth, nausea, fatigue, somnolence, euphoria, vomiting, disorientation, drowsiness, confusion, loss of balance, and hallucination [11]. Cannabis and $\triangle 9$-THC produce dose-related impairments in short-term memory, attention, hand-eye coordination, vigilance, and the perception of time and distance. This profile of impairments may contribute to vehicle and other accidents.

Cannabis can also induce schizophreniform psychosis in normal individuals, precipitate schizophrenia in predisposed individuals, and exacerbate positive symptoms in people with schizophrenia [25]. It remains controversial whether cannabis use alone is a sufficient risk factor for psychosis, or rather a component cause in the pathogenesis [1]. Some studies suggest that in patients with established psychotic disorders, cannabis use may be related to anxiety symptoms, which could exacerbate positive symptoms [26]. As has been shown, such correlations may apply to patients who remain at heightened risk for psychotic disorder, and they have not been evaluated in a prodromal period of illness with prevalent cannabis abuse. Therefore, cannabis use may be a risk factor for exacerbating sub-threshold psychotic symptoms, specifically perceptual disturbances, in high-risk cases [18]. The risks of developing psychotic disorder after the administration of a synthetic cannabinoid remain a matter of debate. Most of the clinical and preclinical data points to a strong correlation between adolescent cannabinoid exposure and their inappropriate use with persistent, adverse neuropsychiatric outcomes in adulthood even after prodromal symptoms of psychosis, parental psychosis, and other substance abuse taken together [20].

Younger people are most vulnerable to altered cognition, emotional functioning, addiction, and risk for psychosis (schizophrenia, bipolar) through early cannabinoid use [3, $18,25]$. The younger the age at intoxication, the poorer the cognitive and psychiatric outcomes in adulthood should be expected. However, definite conclusions cannot yet be made as to whether cannabis use alone negatively impacts upon the human adolescent brain [25].

Chronic use of cannabinoids can result in the development of addiction. In the addiction phenomenon, repeated exposure to drugs of abuse causes hypersensitivity to psychoactive substances and drug-associated stimuli. Sensitisation is defined as the enhancement of a direct drug effect, and it arises through adaptive reactions. An abused drug may have an increased pharmacological effect, for instance due to a rise in the number of receptors or a strengthening of their coupling to effector proteins. Conversely, an increased behavioural effect may be due to the drug's effect on neuronal circuits, with altered patterns of stored information resulting from prior associative learning.

The development of cannabinoid addiction impacts upon several neurobiological processes. It is necessary to analyse how cannabinoids act during initial exposure and after repeated exposure. Much research has focused on the mechanisms by which cannabinoids induce changes in brain function. The changes caused by cannabinoids in brain neuronal circuits, such as neurotransmitters receptors, signalling cascades, and gene expression, have been intensively investigated. Since genetic variability may increase the risk of addictive behaviours, exposure to THC may result in neuroadaptation, i.e. an addicted state. This adaptation occurs at the cellular, molecular, or genetic level and is associated with synaptic plasticity and altered gene expression due to epigenetic processes.

The developments in cellular and molecular neurobiology have focused on how cannabinoids produce persistent alterations in brain function and how they may facilitate the development of drug dependence. Neuronal circuits and their elements are significantly adapted in response to exposure to cannabinoids. THC acts via presynaptic $\mathrm{CB} 1$ receptors to inhibit the release of GABA and glutamate and cause long-term forms of synaptic plasticity by modulation of the number and strength of connections between the neurons [27]. Due to prolonged THC exposure, uncoupling and downregulation of brain CB1 receptors play a crucial role in developing cannabinoids addiction and tolerance.

The addictive potential of cannabis varies considerably, especially in younger people. According to the American Psychiatric Association (APA), a diagnosis of probable cannabis addiction is termed 'cannabis use disorder' (CUD). In this group, the risk of CUD is more frequent, ranging from 33\% to 50\% of individuals who are regularly intoxicated. THC is thought to be the primary cannabis responsible for pharmacodynamic tolerance development up to psychic and physical addiction. At the same time, CBD might be the one to be used in addiction therapy. Further exploration is also needed to understand whether enhanced sensitivity to the cognitive effects of $\Delta^{9}$-THC depends on brain cannabinoid receptor dysfunction, and how these changes contribute to the cognitive deterioration and core pathophysiology symptoms associated with schizophrenia or other neurological and somatoform disorders.

\section{Summary}

There is substantial evidence for the benefits of cannabis or cannabinoids for chronic pain, chemotherapy-induced nausea and vomiting, and patient-reported symptoms of spasticity associated with multiple sclerosis. For some cannabis and cannabinoid indications, the main route of administration studied was the oral route. For chronic pain, most studies have examined oral cannabis extract, although some have examined 
smoked or vaporised cannabis. It is unknown whether and to what degree the results of several studies can be generalised to other products and routes of administration. Many different conditions can influence the potential therapeutic effects of cannabinoids usage.

In several conditions, such as some forms of epilepsy, especially in children, and PTSD, the evaluation of cannabinoids efficacy should be prioritised $[28,29]$ due to the severity of these diseases and the urgent need for new therapeutic approaches. However, the most common limitations are connected with the study design (e.g. a lack of appropriate control groups and/or of long-term follow-ups), small sample sizes, and research gaps in examining the potential therapeutic benefits of different forms of cannabis.

These limitations highlight the need for substantial research to provide comprehensive and conclusive evidence on the therapeutic effects of cannabis and cannabinoids.

Table 2 sets out our elaboration of the results of studies and summarises the quality of the evidence related to the therapeutic use of cannabinoids based on the meta-analyses carried out by the National Academies of Sciences, Engineering, and Medicine; Health and Medicine Division; Board on Population Health and Public Health Practice; Committee on the Health Effects of Marijuana: An Evidence Review and Research Agenda [30].

The medical use of cannabis in Europe refers to the EU Parliament's 2019 resolution, aimed at distinguishing between medical cannabis and cannabis-based medicines. It is suggested that only cannabis-based medicinal products should be considered for medical use [31, 32].

According to the latest literature [33], the European Medicines Agency (EMA) approved only one drug in 2019 for the treatment of intractable childhood epilepsy, while other medicinal products containing other types of cannabinoids were cross-recognised and approved at a national level. However, due to the lack of a uniform approach to the cultivation, manufacturing and use of medical cannabis, a 2018 report by the European Monitoring Centre for Drugs and Drug Addiction (EMCDDA) discussed the various regulatory and legal frameworks of relevance to the medical use of cannabis and cannabinoids. The report presents medicinal products with marketing authorisation available in the EU:

- Cesamet and Canemes (active ingredient - nabilone): oral capsules containing a synthetic cannabinoid similar to THC

- Marinol and Syndros (active ingredient - dronabinol): oral capsules or an oral solution containing synthetic THC (may sometimes refer to plant-derived THC)

- Sativex (active ingredient - nabiximol): sublingual spray containing approximately equal quantities of THC and CBD

- Epidolex (active ingredient - cannabidiol): oral solution containing plant-based CBD.
Other cannabis preparations available in the EU are raw cannabis, magistral preparations, and standardised cannabis preparations including preparations of cannabis flowers, granulates, and oil extracts $[31,32,33]$.

\section{Cannabis and cannabinoids preparations registered and available in Poland}

According to EU regulations and published data [31], in Poland some medicinal products containing cannabinoids should be available, including dronabinol (synthetic $\Delta 9$-THC, capsule), nabilone (synthetic cannabinoid, similar to $\Delta 9$-THC, capsule), and nabiximol (extract of cannabis - $\Delta$ 9-THC and CBD - sublingual spray: Sativex ${ }^{\circledR}$, which has a marketing authorisation). Some of these can be made available to patients through a special permit known as patient import. Sativex ${ }^{\circledast}$ is only available in a few pharmacies in Poland, whereas Cannabidiol is widely available (CBD, oral solution: Epidyolex ${ }^{\circledast}$ ), as it was authorised for use in all EU member states in 2019 as an orphan medicinal product [33].

Listed below are cannabis pharmaceutical products that are available and approved for the Polish market:

- Cannabis extractum normatum THC $10 \%, \mathrm{CBD}<1 \%$ (Cannabis extractum normatum, Pharmaceutical Concern Okoniewscy "Vetos-Farma" Sp. z o.o.)

- Cannabis flos AURORA DEUTSCHLAND GmbH THC $1 \%$, CBD 12\% (Cannabis sativa flos, Aurora Deutschland $\mathrm{GmbH})$

- Cannabis flos AURORA DEUTSCHLAND GmbH THC 20\%, CBD 1\% (Cannabis sativa flos, Aurora Deutschland $\mathrm{GmbH})$

- Cannabis flos AURORA DEUTSCHLAND GmbH THC $22 \%$, CBD 1\% (Cannabis sativa flos, Aurora Deutschland $\mathrm{GmbH})$

- Cannabis flos AURORA DEUTSCHLAND GmbH THC $8 \%$, CBD 8\% (Cannabis sativa flos, Aurora Deutschland $\mathrm{GmbH})$

- Cannabis flos, Canopy Growth THC 10\%, CBD 7\% (Cannabis sativa, Canopy Growth Polska Sp. z o.o.)

- Cannabis sativa L., Red No 2 THC 19\%, CBD 1\% (Cannabis sativa flos, Canopy Growth Polska Sp. z o.o.).

\section{Conclusion}

Although reports concerning the possible medicinal use of cannabis and cannabinoids have raised hopes, we must underline that the current evidence for medical use of these substances seems to be weak, insufficient, or moderate, and they are still under scientific study with limited clinical findings and gaps in documented effects. In Europe, they are used mostly outside the registered indications, as there is still a need for universal drugs with broad use in many neurological diseases, and therefore the decision to use them requires individual analysis. 
Table 2. Analysis of quality of evidence associated with therapeutic use of cannabis and cannabinoids

\section{Statement}

Research evidence

Cannabis is an effective treatment for chronic pain in adults $\rightarrow$ Substantial evidence

Cannabinoids are an effective treatment for cancers, including glioma $\rightarrow$ Insufficient evidence

Oral cannabinoids are effective antiemetics in treatment Conclusive evidence

of chemotherapy-induced nausea and vomiting $\rightarrow$

Cannabis and oral cannabinoids are effective in increasing appetite

Limited evidence

and decreasing weight loss associated with HIV/AIDS $\rightarrow$

Cannabinoids are an effective treatment for cancer-associated anorexia-cachexia syndrome and anorexia nervosa $\rightarrow$

Dronabinol is an effective treatment for symptoms

of irritable bowel syndrome $\rightarrow$

Cannabinoids are an effective treatment for epilepsy $\rightarrow$

Oral cannabinoids are an effective treatment for improving patient-reported multiple sclerosis spasticity symptoms $\rightarrow$

but for effect on clinician-measured spasticity $\rightarrow$

Cannabinoids are an effective treatment for spasticity in patients

with paralysis due to spinal cord injury $\rightarrow$

THC capsules are an effective treatment for improving symptoms

of Tourette's Syndrome $\rightarrow$

Cannabis is an effective treatment for symptoms associated

with amyotrophic lateral sclerosis $\rightarrow$

Oral cannabinoids are an effective treatment for chorea and certain neuropsychiatric symptoms associated with Huntington's Disease $\rightarrow$

Cannabinoids are an effective treatment for motor system symptoms associated with Parkinson's Disease Insufficient evidence or levodopa-induced dyskinesia $\rightarrow$

Nabilone and dronabinol are an effective treatment for dystonia $\rightarrow$

Insufficient evidence

Cannabinoids are ineffective treatment for improving the symptoms associated with dementia $\rightarrow$

Limited evidence

Statistical association between cannabinoids and better outcomes

(i.e. mortality, disability) after traumatic brain injury or intracranial haemorrhage $\rightarrow$

Cannabinoids are an effective treatment for achieving abstinence

in use of addictive substances $\rightarrow$

Cannabidiol is an effective treatment for improving anxiety symptoms, as assessed by a public speaking test, in individuals with social anxiety disorders $\rightarrow$

Nabiximol, dronabinol, and nabilone are ineffective treatment

for reduction of depressive symptoms in individuals with chronic pain

or multiple sclerosis $\rightarrow$

Cannabinoids are an effective treatment to improve short-term sleep outcomes in individuals with

sleep disturbance associated with obstructive sleep apnoea syndrome, fibromyalgia, chronic pain, and multiple sclerosis $\rightarrow$

Nabilone is effective for improving symptoms

of post-traumatic stress disorder $\rightarrow$

Cannabidiol is an effective treatment for mental health outcomes

in individuals with schizophrenia or schizophreniform psychosis $\rightarrow$

Limited evidence

No evidence

Limited evidence

Limited evidence

Moderate evidence

Source: authors' elaborations 
Our review of the available literature presents the perspectives for cannabis and cannabinoids in medical practice, but it also shows the limitations of their application and gaps in the evidence. The need for additional research and clinical studies is also emphasised: better-designed trials on larger research groups, studies on dosage and drug interactions, and studies with longer-term follow-ups are still required.

\section{Conflicts of interest: None}

Funding: None.

\section{References}

1. Häuser W, Finn DP, Kalso E, et al. European Pain Federation (EFIC) position paper on appropriate use of cannabis-based medicines and medical cannabis for chronic pain management. Eur J Pain. 2018; 22(9): 1547-1564, doi: 10.1002/ejp.1297, indexed in Pubmed: 30074291.

2. Pacher P, Bátkai $S$, Kunos $G$. The endocannabinoid system as an emerging target of pharmacotherapy. Pharmacol Rev. 2006; 58(3): 389-462, doi: 10.1124/pr.58.3.2, indexed in Pubmed: 16968947.

3. Lafaye G, Karila L, Blecha L, et al. Cannabis, cannabinoids, and health. Dialogues Clin Neurosci. 2017; 19(3): 309-316, indexed in Pubmed: 29302228.

4. Pertwee RG, Howlett AC, Abood ME, et al. International Union of Basic and Clinical Pharmacology. LXXIX. Cannabinoid receptors and their ligands: beyond $\mathrm{CB}_{1}$ and $\mathrm{CB}_{2}$. Pharmacol Rev. 2010; 62(4): 588-631, doi: 10.1124/pr.110.003004, indexed in Pubmed: 21079038.

5. Mackie K. Signaling via CNS cannabinoid receptors. Mol Cell Endocrinol. 2008; 286(1-2 Suppl 1): S60-S65, doi: 10.1016/j. mce.2008.01.022, indexed in Pubmed: 18336996.

6. Health Canada. Information for health care professionals: Cannabis (marihuana, marijuana) and the cannabinoids. 2018. https://www. canada.ca/en/health-canada/services/drugs-medication/cannabis/ information-medical-practitioners/information-health-care-professionals-cannabis-cannabinoids (ISBN: 978-0-660-27828-5, last accessed 22/11/2021).

7. Hillard CJ. The Endocannabinoid Signaling System in the CNS: A Primer. Int Rev Neurobiol. 2015; 125: 1-47, doi: 10.1016/ bs.irn.2015.10.001, indexed in Pubmed: 26638763.

8. Compagnucci C, Di Siena S, Bustamante MB, et al. Type-1 (CB1) cannabinoid receptor promotes neuronal differentiation and maturation of neural stem cells. PLoS One. 2013; 8(1): e54271, doi: 10.1371/ journal.pone.0054271, indexed in Pubmed: 23372698.

9. Lu HC, Mackie K. An Introduction to the Endogenous Cannabinoid System. Biol Psychiatry. 2016; 79(7): 516-525, doi: 10.1016/j.biopsych.2015.07.028, indexed in Pubmed: 26698193.

10. Graczyk M, Łukowicz M, Dzierzanowski T. Prospects for the Use of Cannabinoids in Psychiatric Disorders. Front Psychiatry. 2021; 12: 620073, doi: 10.3389/fpsyt.2021.620073, indexed in Pubmed: 33776815.

11. Whiting PF, Wolff RF, Deshpande S, et al. Cannabinoids for Medical Use: A Systematic Review and Meta-analysis. JAMA. 2015; 313(24): 2456-2473, doi: 10.1001/jama.2015.6358, indexed in Pubmed: 26103030.

12. Hejazi N, Zhou C, Oz M, et al. Delta9-tetrahydrocannabinol and endogenous cannabinoid anandamide directly potentiate the function of glycine receptors. Mol Pharmacol. 2006; 69(3): 991-997, doi: 10.1124/mol.105.019174, indexed in Pubmed: 16332990.
13. Izzo AA, Borrelli F, Capasso R, et al. Non-psychotropic plant cannabinoids: new therapeutic opportunities from an ancient herb. Trends Pharmacol Sci. 2009; 30(10): 515-527, doi: 10.1016/j. tips.2009.07.006, indexed in Pubmed: 19729208.

14. Di Marzo V, Stella N, Zimmer A. Endocannabinoid signalling and the deteriorating brain. Nat Rev Neurosci. 2015; 16(1): 30-42, doi: 10.1038/nrn3876, indexed in Pubmed: 25524120.

15. Trezza V, Campolongo P. The endocannabinoid system as a possible target to treat both the cognitive and emotional features of post-traumatic stress disorder (PTSD). Front Behav Neurosci. 2013; 7: 100, doi: 10.3389/fnbeh.2013.00100, indexed in Pubmed: 23950739.

16. Silvestri C, Di Marzo V. The endocannabinoid system in energy homeostasis and the etiopathology of metabolic disorders. Cell Metab. 2013; 17(4): 475-490, doi: 10.1016/j.cmet.2013.03.001, indexed in Pubmed: 23562074.

17. Curtis A, Clarke CE, Rickards HE. Cannabinoids for Tourette's Syndrome. Cochrane Database Syst Rev. 2009(4): CD006565, doi: 10.1002/14651858.CD006565.pub2, indexed in Pubmed: 19821373

18. Corcoran CM, Kimhy D, Stanford A, et al. Temporal association of cannabis use with symptoms in individuals at clinical high risk for psychosis. Schizophr Res. 2008; 106(2-3): 286-293, doi: 10.1016/j. schres.2008.08.008, indexed in Pubmed: 18809298.

19. Johnson JR, Burnell-Nugent M, Lossignol D, et al. Multicenter, double-blind, randomized, placebo-controlled, parallel-group study of the efficacy, safety, and tolerability of THC:CBD extract and THC extract in patients with intractable cancer-related pain. J Pain Symptom Manage. 2010; 39(2): 167-179, doi: 10.1016/j.jpainsymman.2009.06.008, indexed in Pubmed: 19896326.

20. Pertwee RG. Receptors and channels targeted by synthetic cannabinoid receptor agonists and antagonists. Curr Med Chem. 2010; 17(14): 1360-1381, doi: 10.2174/092986710790980050, indexed in Pubmed: 20166927.

21. Fantegrossi WE, Wilson CD, Berquist MD. Pro-psychotic effects of synthetic cannabinoids: interactions with central dopamine, serotonin, and glutamate systems. Drug Metab Rev. 2018; 50(1): 65-73, doi: 10.1080/03602532.2018.1428343, indexed in Pubmed: 29385930.

22. Katona I, Freund TF. Multiple functions of endocannabinoid signaling in the brain. Annu Rev Neurosci. 2012; 35: 529-558, doi: 10.1146/ annurev-neuro-062111-150420, indexed in Pubmed: 22524785.

23. Lutz B, Marsicano G, Maldonado R, et al. The endocannabinoid system in guarding against fear, anxiety and stress. Nat Rev Neurosci. 2015; 16(12): 705-718, doi: 10.1038/nrn4036, indexed in Pubmed: 26585799

24. Basavarajappa BS. Neuropharmacology of the endocannabinoid signaling system-molecular mechanisms, biological actions and synaptic plasticity. Curr Neuropharmacol. 2007; 5(2): 81-97, doi: 10.2174/157015907780866910, indexed in Pubmed: 18084639.

25. Levine A, Clemenza K, Rynn M, et al. Evidence for the Risks and Consequences of Adolescent Cannabis Exposure. J Am Acad Child Adolesc Psychiatry. 2017; 56(3): 214-225, doi: 10.1016/j.jaac.2016.12.014, indexed in Pubmed: 28219487.

26. Mustonen A, Niemelä S, Nordström T, et al. Adolescent cannabis use, baseline prodromal symptoms and the risk of psychosis. Br J Psychiatry. 2018; 212(4): 227-233, doi: 10.1192/bjp.2017.52, indexed in Pubmed: 29557758.

27. Fratta W, Fattore L. Molecular mechanisms of cannabinoid addiction. Curr Opin Neurobiol. 2013; 23(4): 487-492, doi: 10.1016/j. conb. 2013.02.002, indexed in Pubmed: 23490548. 
28. Cougle JR, Bonn-Miller MO, Vujanovic AA, et al. Posttraumatic stress disorder and cannabis use in a nationally representative sample. Psychol Addict Behav. 2011; 25(3): 554-558, doi: 10.1037/a0023076, indexed in Pubmed: 21480682.

29. Massot-Tarrús A, McLachlan RS. Marijuana use in adults admitted to a Canadian epilepsy monitoring unit. Epilepsy Behav. 2016; 63: 73-78, doi: 10.1016/j.yebeh.2016.08.002, indexed in Pubmed: 27568641.

30. National Academies of Sciences, Engineering, and Medicine; Health and Medicine Division; Board on Population Health and Public Health Practice; Committee on the Health Effects of Marijuana: An Evidence Review and Research Agenda. The Health Effects of Cannabis and Cannabinoids: The Current State of Evidence and Recommendations for Research. Washington (DC): National Academies Press (US); 2017 Jan 12. PMID: 28182367. Therapeutic Effects of Canna- bis and Cannabinoids. https://pubmed.ncbi.nlm.nih.gov/28182367/ (22/11/2021).

31. European Monitoring Centre for Drugs and Drugs Addiction (2018), Medical use of cannabis and cannabinoids: questions and answers for policymaking, Publication Office of the European Union, Luxembourg. https://www.emcdda.europa.eu/publications/rapid-communications/medical-use-of-cannabis-and-cannabinoids-questions-and-answers-for-policymaking_en (17/01/2022).

32. Medical Cannabis in Europe. European Industrial Pharmacists Group (EIPG). https://epig.eu/medical-cannabis-in-europe/ (17/01/2022).

33. Lipnik-Štangelj M, Razinger B. A regulatory take on cannabis and cannabinoids for medicinal use in the European Union. Arh Hig Rada Toksikol. 2020; 71(1): 12-18, doi: 10.2478/aiht-2020-71-3302, indexed in Pubmed: 32597142. 